\title{
Surgery for infants with a hypoplastic systemic ventricle and severe outflow obstruction: early results with a modified Norwood procedure
}

F A Bu'Lock, O Stümper, R Jagtap, E D Silove, J V De Giovanni, J G C Wright, B Sethia, W J Brawn

\begin{abstract}
Objective-Prospective audit of the first year of implementation of a modified approach to palliation for infants with hypoplastic systemic ventricle and severe systemic outflow obstruction.
\end{abstract}

Setting-Tertiary referral centre for neonatal and infant cardiac surgery.

Patients and methods-17 of 19 infants (aged < 35 days) presenting to Birmingham Children's Hospital in 1993 with hypoplastic systemic ventricle and severe outflow obstruction underwent surgery. This was performed using a new modification of the Norwood-type arch repair, without the use of exogenous material, and a $3.5 \mathrm{~mm}$ Gore-tex shunt between the innominate and right pulmonary arteries. The Gore-tex shunt was replaced by a cavopulmonary shunt between 3 and 5 months later. Clinical, morphological, and functional determinants of outcome were examined.

Results-10 (59\%) infants survived initial surgery. All proceeded to cavopulmonary shunt without further loss. Significant atrioventricular valve regurgitation seemed to be the main risk factor for poor outcome. If this was excluded, the morphology of the dominant ventricle seemed to have little effect on the outcome of initial surgery.

Conclusions-Early survival was achieved in $59 \%$ of patients in the first year of implementation of a protocol for surgery in infants with hypoplastic systemic ventricle and severe outflow obstruction. The construction of a neoaorta without the use of exogenous material may allow improved later growth of the neoaorta. Early cavopulmonary shunt can be performed safely and should reduce mid-term complications from cyanosis and systemic ventricular volume loading.

(Br Heart f 1995;73:456-461)

Keywords: Norwood procedure; hypoplastic systemic ventricle; palliation

Infants with a hypoplastic systemic ventricle and severe outflow obstruction have a duct dependent systemic circulation and will die without early intervention, irrespective of precise cardiac morphology. Staged palliative surgery and heart transplantation for hypoplastic left heart syndrome and functionally similar conditions have now been successfully practised in a number of centres in the USA for several years, with current mediumterm survival of more than $80 \% .^{12}$ So far, however, these results have not been matched in the UK and most centres still propose terminal supportive care ${ }^{3}$ as the first line management for infants with these conditions.

In the years up to December 1992, 21 infants with a hypoplastic systemic ventricle and severe outflow tract obstruction, whose parents felt strongly that they should be offered any possible option of longer term survival, underwent surgery at Birmingham Children's Hospital. Fourteen infants had hypoplastic left heart syndrome with severe stenosis or atresia either or both of the mitral and aortic valves and hypoplasia of the left ventricle and aortic arch and seven had functionally similar conditions but with a hypoplastic right and dominant left ventricle. Of five $(24 \%)$ infants who survived (mean (range) follow up $2 \cdot 4(1 \cdot 75-3 \cdot 25)$ years) two have proceeded to fenestrated Fontan's procedure and three have undergone cavopulmonary shunt and await further surgery. Six patients underwent initial bilateral pulmonary artery banding (with an aortopulmonary shunt or ductal patency maintained on prostaglandin $\mathrm{E}_{2}$ ) with a view to later pulmonary artery to aortic anastomosis and cavopulmonary shunt. Two of these infants survived, having recently undergone completion of Fontan's operation and are the only survivors before August 1992. None of the 10 infants undergoing early Norwood-type repair, ${ }^{4}$ with a variety of types and sizes of systemic to pulmonary artery connection, survived, although one child lived for 10 months. The other three survivors were from a group of five infants who underwent a new modification of the Norwood operation, first practised in Birmingham in August 1992, in which the aortic arch is repaired without the use of exogenous material and a $3.5 \mathrm{~mm}$ Gore-tex shunt is placed between the innominate and right pulmonary arteries. All three have subsequently successfully undergone superior cavopulmonary anastomosis and take down of the Gore-tex shunt.

Combined with increasing experience of postoperative management, this new modification of the Norwood technique seemed to offer more promise than previous approaches. From January 1993 it was therefore decided to consider such surgery for all infants presenting with hypoplastic systemic ventricle 
and severe outflow obstruction to Birmingham Children's Hospital. A prospective audit of this policy has been carried out, in an attempt to speed up the learning curve and to optimise the management of this challenging condition. Experience of the first year of such a policy is described here, with reference to previous experience and ventricular morphology.

\section{Patients and methods}

STUDY GROUP

Between 1 January and 31 December 1993, 19 infants with hypoplastic systemic ventricle and severe outflow obstruction presented to Birmingham Children's Hospital. Surgery was discussed in all cases, but after careful counselling, the parents of two infants declined surgery and both died soon afterwards. Thus 17 infants underwent surgery. There were four girls and 13 boys, aged between $5 \mathrm{~h}$ and 34 (mean 9.6) days on arrival at our unit, weighing between 2.6 and 4.4 (mean 3.5 ) $\mathrm{kg}$. The majority (16 of 17 ) were term infants (>37 weeks gestational age). Two were infants of diabetic mothers of whom one was born at 34 weeks' gestation weighing $4.2 \mathrm{~kg}$. Only four infants had congenital heart disease detected antenatally, and two had mothers with poor obstetric histories. Thirteen infants $(76 \%)$ arrived in good condition; nine on prostaglandin $E_{2}$ infusions and four on no medication. Three were acidotic on arrival and were not receiving prostaglandin. One patient arrived "in extremis", acidotic despite prostaglandin, inotropes, ventilation, and peritoneal dialysis.

\section{ECHOCARDIOGRAPHIC ASSESSMENT}

All patients underwent detailed echocardiographic examination before surgery. Echocardiographic data were recorded on standard diagnostic equipment from the usual precordial windows and cardiac anatomy defined according to standard criteria.

Left and right ventricular length were measured from the apical four chamber view between the apical endocardium and the crux of the heart within the respective ventricles. Maximum ventricular diameters were measured at the approximate level of the chordae tendinae and maximum ventricular areas were measured by planimetry from the same view. In addition, mitral and tricuspid valve diameters were measured on the ventricular endocardial surface, between the hinge points of the leaflets where these were considered to be present. The maximum diameter of the atrial septal defect was measured from the subcostal window.

The diameters of the aortic valve and ascending aorta were measured from parasternal long axis views. Aortic valve diameter was taken at the position of the apparent hinge points of the valve leaflets, ascending aortic diameter above the level of the sinuses of Valsalva. The diameter of the transverse arch was measured between the head and neck vessels from the suprasternal view, along with the descending aortic diameter distal to any coarctation noted.

The degree of any atrioventricular valve regurgitation detectable by colour flow Doppler echocardiography was graded from 1 to 3. Grade 1 represented a trivial closing jet close to the valve. In grade 2 regurgitation the regurgitant jet extended up to half way back into or around the side of the atrium and grade 3 regurgitation required a broader jet extending more than half way back into the atrium.

\section{PREOPERATIVE MANAGEMENT}

All patients were stabilised as much as possible before surgery, using intravenous prostaglandin infusion, correction of acid base status, ventilation, and inotropes as required. Infants were maintained in as low an inspired oxygen concentration as possible (usually air), and arterial partial pressure of carbon dioxide $\left(\mathrm{PCO}_{2}\right)$ was monitored and kept $>4.5 \mathrm{kPa}$.

\section{OPERATIVE TECHNIQUE}

Surgery was performed on cardiopulmonary bypass with cold St Thomas' crystalloid cardioplegic arrest, hypothermia (minimum $18^{\circ} \mathrm{C}$ nasopharyngeal), and periods of complete circulatory arrest. Under circulatory arrest the arch vessels were mobilised extensively and cross clamped. All ductal tissue was excised, including the segment of aorta where the coarctation is normally found, even when no clear coarctation was present (figure). The main pulmonary artery was transected just proximal to the bifurcation and the distal pulmonary arteries were repaired with a patch of native or bovine pericardium. The descending thoracic aorta was widely mobilised by blunt dissection, without division of intercostal arteries. The aortic arch was opened inferiorly back down into the ascending aorta to the level of the transected end of the main pulmonary artery, with mobilisation of the ascending aorta to the same level. The back wall of the descending thoracic aorta was anastomosed to the posterior wall of the aortic arch without undue tension, avoiding compression of the left pulmonary artery. The proximal main pulmonary artery was anastomosed to this confluence, extending the anastomosis down to the opened out ascending aorta. An open atrial septectomy was performed and the distal anastomosis of the 3.5 mm Gore-tex shunt to the upper border of the right pulmonary artery was created. The heart was then recannulated, deaired, clamps removed, and the body and heart rewarmed and reperfused. The anastomosis of the 3.5 $\mathrm{mm}$ Gore-tex shunt to the innominate artery was performed with a side biting clamp applied to the innominate artery for haemostasis. Continuous $7 \cdot 0$ prolene was used for all anastomoses and interrupted sutures were not used.

\section{POSTOPERATIVE MANAGEMENT}

Patients were initially managed with adrenaline, dobutamine, dopamine, and sodium nitroprusside as required in the intensive care 
Diagrammatic representation of the aortic arch anatomy and repair without the use of exogenous material. All ductal tissue (crosshatched), including the segment of aorta where the coarctation is normally found, is excised. The main pulmonary artery (MPA) is disconnected from the branch pulmonary arteries and, with the descending aorta, anastomosed directly to the underside of the aortic arch. The pulmonary arteries are repaired with pericardium and a $3.5 \mathrm{~mm}$ Gore-tex shunt is placed between the innominate and proximal right pulmonary arteries. An open atrial septectomy is also performed.
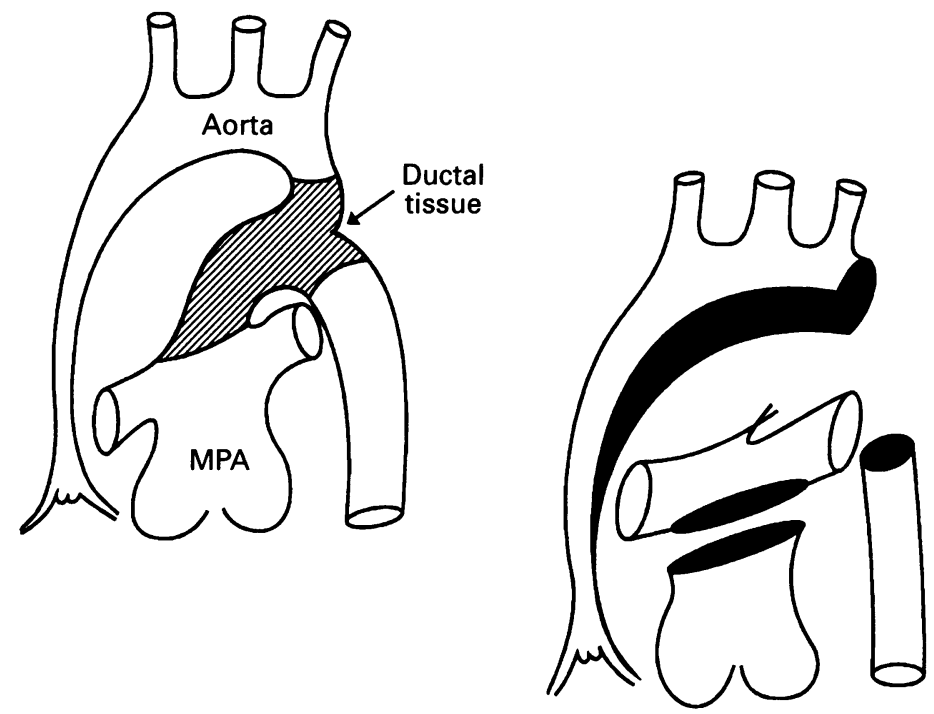

$3.5 \mathrm{~mm}$ Gore-tex shunt

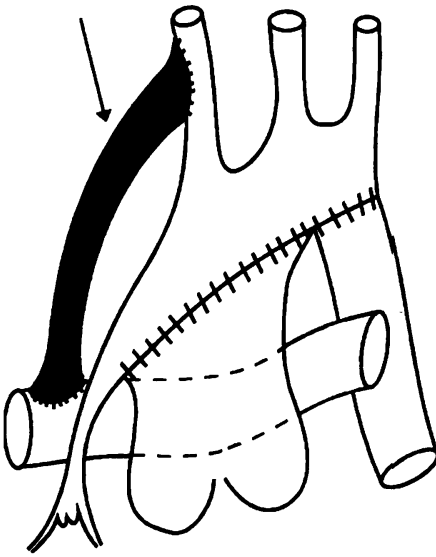

unit. Mechanical ventilation was performed using pressure regulated neonatal ventilators. Inspired oxygen concentration was adjusted as necessary to maintain arterial oxygen saturations of $70-80 \%$, ventilation rates and pressures being adjusted to maintain the arterial $\mathrm{PCO}_{2}$ between 4.5 and $6 \mathrm{kPa}$.

Delayed chest closure was performed when the infants were haemodynamically stable. Patients were transferred to the infant cardiac ward as soon as deemed fit after extubation, residual inotropic support (dobutamine) often being withdrawn several days later. Patients with Gore-tex shunts were discharged on aspirin $(5 \mathrm{mg} / \mathrm{kg})$ with appropriate antifailure medication as required.

LATE MANAGEMENT AND FURTHER SURGERY A policy of early cardiac catheterisation and conversion to cavopulmonary shunt was practised because of the relatively small shunt size $(3.5 \mathrm{~mm})$.

Elective cardiac catheterisation was performed at 3-5 months of age under general anaesthesia, neuromuscular blockade, and full ventilation. A percutaneous femoral venous approach was used for assessment of the intracardiac circulation, with anterograde aortic cannulation where possible. Balloon angioplasty of postoperative arch stenoses was performed when indicated.

Superior cavopulmonary connection and take-down of the Gore-tex shunt was then performed via a midline sternotomy on cardiopulmonary bypass or circulatory arrest, or both, with revision of the neoaorta or branch pulmonary arteries as necessary. Postoperative management was as standard for cavopulmonary shunts in our unit, with early extubation and head elevation.

\section{Results}

INITIAL SURGERY

It was possible to perform the new modification of the Norwood arch repair without exogenous material in 16 of 17 patients. A pulmonary homograft was used in the patient with congenitally corrected transposition of the great vessels to augment the arch repair in view of the very anterior position of the ascending aorta.

Surgery was performed a mean of $2 \cdot 7$ days from presentation. One patient had undergone coarctation repair as the intended primary procedure but had failed to sustain adequate cardiac output and underwent Norwood-type surgery on day 23 after arrival.

\section{SHORT-TERM OUTCOME}

Of the 17 infants who underwent surgery, three could not be weaned from cardiopulmonary bypass and died in the operating theatre. Thus $14(82 \%)$ returned to the intensive care unit (operative survivors). There were two further deaths associated with an unrecoverable low output state within $12 \mathrm{~h}$ of surgery. Two patients died later, on postoperative days 14 and 35 respectively. Each infant collapsed with abdominal distension and suspected "necrotising enterocolitis" (one also had postoperative paraplegia). Thus 10 infants $(59 \%)$ survived initial surgery.

Excluding those patients who were unable to be weaned from cardiopulmonary support, mean (range) bypass time was 53 (40-182) min, with mean (range) cross clamp time of 57 (46-65) $\mathrm{min}$ and mean (range) cardiac

Postoperative artificial ventilation was required for a mean (range) of 5.5 (2-12) days in 11 of the 12 patients who survived to arrest time of $51(40-87) \mathrm{min}$. 
be extubated. One patient, having been extremely sick before operation, still required full ventilation 19 days after initial surgery. It was considered that excessive pulmonary blood flow might be contributing and the Gore-tex shunt was taken down and replaced by a cavopulmonary shunt on postoperative day 19 . This child was subsequently successfully extubated 8 days later. Three patients required postoperative peritoneal dialysis (one continued from before operation) and one of these had prolonged jaundice, probably related to neonatal/ischaemic hepatitis, which has since resolved. Mean postoperative stay was 19 days, being between 10 and 26 days except for one patient who had a protracted admission (52 days) because of severe gastrooesophageal reflux.

\section{MEDIUM-TERM OUTCOME AND}

CAVOPULMONARY SHUNT

There has been no "out of hospital" mortality so far, and all survivors of initial surgery have now successfully undergone cavopulmonary shunt.

As discussed earlier, one patient underwent cavopulmonary shunt 19 days after initial surgery. The remaining nine underwent cardiac catheterisation between 3 and 5.5 (mean $4 \cdot 1)$ months of age. Three patients had mild narrowing of the transverse to descending aortic anastomosis, with gradients $<10 \mathrm{~mm} \mathrm{Hg}$, which were dealt with by release and resection of fibrous tissue at the time of surgery. One patient had more significant recoarctation with a gradient of $25 \mathrm{~mm} \mathrm{Hg}$. This was treated with balloon angioplasty using a $7 \mathrm{~mm}$ balloon catheter and the gradient was reduced to $8 \mathrm{~mm} \mathrm{Hg}$. There were mild narrowings of the left or both branch pulmonary arteries seen on angiography (the shunt was not crossed) in five patients, but only two required patch augmentation at surgery, the remainder were dealt with by release and resection of fibrous tissue.

There have been no deaths, patients were extubated $<12 \mathrm{~h}$ after operation, and all had a relatively short postoperative hospital stay (mean (range) ten (6-13) days). Recovery was complicated in one child by a hydrothorax and fluid retention. Another child required readmission for a chylopericardium and eventually required surgical creation of a pericardial window.

The 10 survivors are well, at home, on low dose diuretics and captopril, and all have peripheral oxygen saturations of $>75 \%$. No patient has significant neoaortic regurgitation, although several have a trivial closing jet detectable on colour Doppler echocardiography.

\section{FACTORS INFLUENCING OUTCOME}

Clinical and perioperative features

The condition of the infant at presentation had no relation to outcome in our series because of the four infants who were acidotic on arrival, two died and two survived, including the child in the worst condition. Similarly, there was no effect of age at presentation or time between presentation and surgery, although apart from the patient who initially underwent coarctation repair, there was little variation in time to surgery. Patients generally underwent surgery as soon as feasible after optimum stabilisation had been achieved. Two of the four infants in whom heart disease was diagnosed antenatally died.

Numbers are too small to draw meaningful conclusions from examination of any relation between duration of intraoperative support and outcome. No patient required additional inspired $\mathrm{CO}_{2}$ to maintain arterial $\mathrm{PCO}_{2}$ between 4.5 and $6.0 \mathrm{kPa}$. A requirement for postoperative dialysis did not appear to adversely affect outcome; only one of the three patients dialysed died, and that was a much later event.

\section{Echocardiographic anatomy and ventricular morphology}

All patients had an atrial septal defect and patent arterial duct. Twelve infants had hypoplastic left and dominant right ventricles. Seven of these had hypoplastic left heart syndrome, three with mitral and aortic atresia and four with a patent but stenotic mitral valve, of whom three had patent aortic valves and one had subaortic atresia. Two infants had atrioventricular septal defects with mitral and aortic stenosis or atresia. One had no ventricular component to the atrioventricular septal defect, in the other the ventricular septal defect was very small. Three infants had mitral stenosis or atresia associated with a malaligned ventricular septal defect or double outlet right ventricle, with subaortic stenosis related to the ventricular septal defect. Nine infants had coarctation detectable on echocardiography. The three infants in whom no coarctation was seen were $<24 \mathrm{~h}$ old with a widely patent arterial duct.

Five patients had hypoplastic right and dominant left ventricles. Two of these had double inlet left ventricle with mitral stenosis, transposition of the great vessels, and subaortic stenosis due to a restrictive ventricular septal defect. One of these infants also had subpulmonary stenosis from accessory atrioventricular valve tissue. Two patients had tricuspid atresia with transposition of the great vessels and subaortic stenosis, again related to a restrictive ventricular septal defect. One child had atrioventricular and ventriculoarterial discordance, with left atrioventricular valve atresia and subaortic stenosis. All five patients had coarctation detectable echocardiographically.

Six $(50 \%)$ of the 12 infants with a right ventricular dependent circulation survived compared with four of five $(80 \%)$ with a left ventricular dependent circulation.

Tricuspid regurgitation was detectable by colour Doppler echocardiography in all patients with a dominant right ventricle while mitral regurgitation was seen only in one of the five with a dominant left ventricle. None of the three infants with more than mild atrioventricular valve regurgitation survived (one had grade 2 and two had grade 3 tricuspid regurgitation) compared with nine of the 14 with absent or grade 1 regurgitation. 
Table 1 Echocardiographic dimensions

\begin{tabular}{|c|c|c|c|c|c|c|c|c|c|c|c|}
\hline & $\begin{array}{l}M V D^{*} \\
(\mathrm{~cm})\end{array}$ & $\begin{array}{l}L V L \\
(\mathrm{~cm})\end{array}$ & $\begin{array}{l}L V D \\
(\mathrm{~cm})\end{array}$ & $\begin{array}{l}L V A \\
\left(\mathrm{~cm}^{2}\right)\end{array}$ & $\begin{array}{l}T V D \\
(\mathrm{~cm})\end{array}$ & $\begin{array}{l}R V L \\
(\mathrm{~cm})\end{array}$ & $\begin{array}{l}R V W \\
(\mathrm{~cm})\end{array}$ & $\begin{array}{l}R V A \\
\left(\mathrm{~cm}^{2}\right)\end{array}$ & $\begin{array}{c}A S D \\
(\mathrm{~cm})\end{array}$ & $\begin{array}{l}W t \\
(\mathrm{~kg})\end{array}$ & $n$ \\
\hline $\begin{array}{l}\text { Dominant RV } \\
\text { Dominant LV }\end{array}$ & $\begin{array}{l}0 \cdot 92 \\
(0 \cdot 6-1 \cdot 6) \\
1 \cdot 27 \\
(0 \cdot 6-1 \cdot 77)\end{array}$ & $\begin{array}{l}2 \cdot 1 \\
(1 \cdot 1-3 \cdot 16) \\
3 \cdot 37 \\
(2 \cdot 38-4 \cdot 53)\end{array}$ & $\begin{array}{l}0.97 \\
(0 \cdot 61-1 \cdot 3) \\
2 \cdot 31 \\
(1 \cdot 78-2 \cdot 73)\end{array}$ & $\begin{array}{l}1 \cdot 92 \\
(0 \cdot 57-3 \cdot 76) \\
6 \cdot 68 \\
(4 \cdot 38-9 \cdot 86)\end{array}$ & $\begin{array}{l}1.93 \\
(1.5-2 \cdot 57) \\
\star \star\end{array}$ & $\begin{array}{l}2 \cdot 33 \\
(2 \cdot 35-4 \cdot 3) \\
1 \cdot 52 \\
(1 \cdot 3-1 \cdot 7)\end{array}$ & $\begin{array}{l}2 \cdot 18 \\
(1 \cdot 56-3 \cdot 32) \\
0 \cdot 67 \\
(0 \cdot 58-0 \cdot 83)\end{array}$ & $\begin{array}{l}6.06 \\
(3.98-10.4) \\
0.91 \\
(0.82-1.06)\end{array}$ & $\begin{array}{l}0.78 \\
(0.52-2 \cdot 4) \\
0.65 \\
(0 \cdot 42-1 \cdot 26)\end{array}$ & $\begin{array}{l}3 \cdot 51 \\
(2 \cdot 58-4 \cdot 4) \\
3 \cdot 59 \\
(2 \cdot 7-4 \cdot 2)\end{array}$ & $\begin{array}{r}12 \\
5\end{array}$ \\
\hline
\end{tabular}

Values are mean (range)

$\star^{\star}$ Mean of 10 patients with valves present (two imperforate). Two patients had no mitral valve.

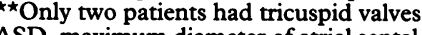

ASD, maximum diameter of atrial septal defect; LV, left ventricle; LVL, maximum LV length from apical endocardium to LV endocardial surface of crux; LVD, maxmum LV diameter at position of chordae tendinae; LVA, maximum LV area measured by planimetry; MVD, mitral valve diameter measured between LV endocardial surfaces of hinge points; RV, right ventricle; RVL, maximum RV length from apical endocardium to RV endocardial surface of crux; RV, maximum RV diameter at position of chordae tendineae; RVA, maximum RV area measured by planimetry; TVD, tricuspid valve diameter measured between RV endocardial surfaces of hinge points; $\mathrm{Wt}$, weight.

Table 1 gives the cardiac dimensions measured echocardiographically, subdivided by dominant ventricular morphology. True hypoplasia of the non-dominant ventricle is well demonstrated compared with the dimensions of the same ventricle for the opposite morphological group. Infants in both groups were of similar weight and there was no difference in the size of the interatrial communication (Mann-Whitney $U$ test). Mitral valve dimensions were similar in both groups $(P=$ 0.14 ) which highlights the fact that valve annulus, not orifice diameter was measured; two of these valves were imperforate. There was some overlap in left ventricle length between the groups, reflecting the "slit-like" ventricles seen in some infants with hypoplastic left heart syndrome, but left ventricle length was still significantly subnormal and less than in the left ventricle dominant group $(P=0.02)$. There was no overlap between groups for other left or right ventricular dimensions $(P=0.002)$. There was no apparent relation between the size of either the dominant or non-dominant ventricle and outcome in either group. Similarly, there was no relation between the ratio of atrial septal defect to dominant atrioventricular valve dimension and mortality.

Table 2 shows aortic dimensions, again subdivided by ventricular morphology. Although aortic valve dimension was significantly larger in those with a dominant left ventricle $(P=0.005)$, the degree of hypoplasia of the rest of the arch and the incidence of coarctation were similar in each group. The precise site and severity of arch hypoplasia varied markedly between individuals and did not seem to be related to either ventricular morphology or outcome.

Table 2 Aortic dimensions

\begin{tabular}{llllllll}
\hline & $\begin{array}{l}\text { AoV } \\
(\mathrm{cm})\end{array}$ & $\begin{array}{l}\text { Asc Ao } \\
(\mathrm{cm})\end{array}$ & $\begin{array}{l}\text { T Arch } \\
(\mathrm{cm})\end{array}$ & $\begin{array}{l}\text { Desc Ao } \\
\left(\mathrm{cm}^{2}\right)\end{array}$ & $\begin{array}{l}\text { Coarct } \\
\text { (Y/N) }\end{array}$ & $\begin{array}{l}W t \\
(\mathrm{~kg})\end{array}$ & $n$ \\
\hline Dominant RV & 0.46 & 0.57 & 0.47 & 0.59 & $9 / 3$ & 3.51 & 12 \\
& $(0.25-071)$ & $(0.24-0.74)$ & $(0.23-0.84)$ & $0.45-0.78)$ & & $(2.58-4.4)$ \\
Dominant LV & 0.78 & 0.58 & 0.40 & 0.60 & $5 / 0$ & 3.59 & 5 \\
& $(0.52-0.91)$ & $(0.5-0.7)$ & $(0.31-0.6)$ & $(0.52-0.71)$ & & $(2.7-4.2)$ & \\
\hline
\end{tabular}

Values are mean (range).

AoV, aortic valve diameter between hinge points of leaflets; Asc Ao, diameter of ascending aorta above sinuses of Valsalva; Coarct $(\mathrm{Y} / \mathrm{N})$, number of patients with coarctation present $(\mathrm{Y})$ or absent $(N)$ on echocardiography; Desc Ao, diameter of descending aorta distal to coarctation site; LV, left ventricle; RV, right ventricle; T Arch, diameter of transverse arch between head and neck vessels; Wt, weight.

\section{Discussion}

We have presented a prospective audit of the first year of a policy of offering a new type of palliative surgery for infants with hypoplastic systemic ventricle and severe outflow obstruction. Several important points have been highlighted in the results section and merit further discussion.

A new type of aortic arch reconstruction using the main pulmonary artery and descending aorta alone to augment the transverse arch is clearly possible. This obviates the need for arch augmentation with exogenous tissue in many cases, which may be beneficial for future growth of the neoaorta, although follow up is currently too short to evaluate this accurately. The incidence of neoaortic valve regurgitation is, so far, negligible with this type of repair, and as yet, only one patient has required balloon angioplasty for recoarctation. If balloon angioplasty is required, the absence of exogenous material in the arch repair might, at least in theory, also render angioplasty safer.

The use of a $3.5 \mathrm{~mm}$ Gore-tex shunt seemed to be highly beneficial to survival from our pre-1993 experience, although its introduction coincided with modifications to the arch repair. The balance between systemic and pulmonary blood flow is critical for early survival of these infants ${ }^{5-7}$ and increased experience in the manipulation of systemic and pulmonary vascular resistance by a dedicated intensive care unit team has undoubtedly also made a major contribution to our improved results. The use of specialised neonatal ventilators may also explain the ability to regulate the arterial $\mathrm{PCO}_{2}$ by ventilation alone, without the use of additional inspired $\mathrm{CO}_{2}$.

Operative mortality for 1993 was $41 \%$, which is significantly better than previously and is likely to improve further with better selection criteria and further increase in experience. Risk factors for mortality are similar to those of other much larger series ${ }^{8-10}$; the effect of significant dominant atrioventricular valve regurgitation being particularly highlighted in this small group. If the three patients with grade 2 or 3 tricuspid regurgitation (two of whom had overtly dysplastic valves) are excluded, six $(67 \%)$ of nine infants with a dominant right ventricle survived against four $(80 \%)$ of five with a dominant left ventricle. Although only two (14\%) of the 14 pre-1993 patients with hypoplastic left heart syndrome 
survived as opposed to three (43\%) of seven with left ventricle dominant circulation, the effects of the learning curve and also of different surgical techniques make it difficult to interpret any comparison in outcome. From our 1993 experience it seems that if infants with unsuitable tricuspid valve anatomy and function are not offered surgery, there is little difference in early outcome between patients with a dominant right or left ventricular morphology. These findings are in broad agreement with those of the Philadelphia experience. ${ }^{11}$ Patient selection criteria continue to change and in fact one of the patients whose parents declined surgery had a severely regurgitant and dysplastic tricuspid valve and counselling was heavily weighted against surgery in this child. It is difficult to comment on factors such as condition at presentation and time to surgery in this small series, but clinical experience supports the need for early surgery once adequate stabilisation has been achieved. The lack of influence of aortic arch anatomy and dimensions on survival is, perhaps, also noteworthy.

Morbidity patterns are also similar to those reported in other series. ${ }^{12-14}$ Postoperative paraplegia in the patient with double inlet left ventricle is an unusual but recognised complication of coarctation repair; perioperative spinal ischaemic insult may have been compounded by preoperative hypoglycaemia in this preterm (34 weeks' gestation) infant of a diabetic mother. Mesenteric ischaemia and colitis is a well recognised and usually lethal complication of the Norwood procedure..$^{15}$

Although the use of a $3.5 \mathrm{~mm}$ Gore-tex shunt appears largely to prevent problems from excessive pulmonary blood flow in the initial postoperative period, it is rather small for the older infant and may subsequently lead to excessive cyanosis. It has therefore been our policy to convert all patients to a cavopulmonary shunt ${ }^{16}$ relatively early (3-5 months of age), to avoid problems related to shunt insufficiency or from ventricular volume overload. ${ }^{14}{ }^{17}$ Cardiac catheterisation and further surgery within 3-5 months from initial palliation have so far been extremely well tolerated and also allow early revision of any problems from the primary repair. Good systemic oxygenation is achieved and access to the pulmonary vasculature is improved if further interventional catheterisation is required. It is not yet clear whether infant pulmonary arteries will continue to grow adequately at venous pressures, but at least so far, and from our experience in other lesions, the situation is encouraging.

\section{Conclusions}

Surgery for infants with hypoplastic systemic ventricle and severe outflow obstruction remains an extremely challenging proposition, demanding much from medical, surgical, and nursing teams alike, as well as from the families of the infants concerned. With an improved surgical technique and a protracted learning period, however, we are now able to offer pal- liative surgery to infants with hypoplastic left heart syndrome and functionally similar conditions with a reasonable prospect of early success.

Repair of the aortic arch can be effected without the use of exogenous material in many cases; the use of early cavopulmonary shunting may prevent complications arising from use of the relatively small $3.5 \mathrm{~mm}$ Goretex shunt at initial surgery. The potential for growth of infant pulmonary arteries at venous pressures remains unknown.

Patients with important abnormalities of the dominant atrioventricular valve should not be offered this type of surgery. If this is borne in mind, results of initial surgery are largely independent of the underlying ventricular morphology in our experience, which is in accordance with those of other series. Results for later completion of Fontan's procedure also seem similar for left and right ventricular dominant anatomy, ${ }^{11} 18$ although whether this remains true in the longer term has to be determined.

The authors thank the referring physicians and all staff of the Heart Unit at Birmingham Children's Hospital, without whom this work would not have been possible.

1 Norwood WI, Jacobs ML, Murphy JD. Fontan procedure for hypoplastic left heart syndrome. Ann Thorac Surg 1992;54:1025-30.

2 Bailey LL, Gundry SR, Razzouk AJ, et al. Bless the babies: One hundred fifteen late survivors of heart transplantation during the first year of life. $\mathcal{F}$ Thorac Cardiovasc Surg 1993;105:805-15.

3 Zakha KG, Spector M, Hanisch D. Hypoplastic left heart syndrome. Norwood operation, transplantation, or compassionate care. Clin Perinatol 1993;20:145-54.

4 Norwood WI, Kirklin JK, Sanders SP. Hypoplastic left heart syndrome: experience with palliative surgery. $A m \mathcal{F}$ Cardiol 1980;45:87-91.

5 Hansen DD, Hickey PR. Anesthesia for hypoplastic left heart syndrome: use of high dose fentanyl in 30 neonates. Anesth Analg 1986;65:127-32.

6 Jonas RA, Lang P, Hansen D, Hickey P, Castaneda AR. First stage palliation of hypoplastic left heart syndrome. The importance of coarctation and shunt size. F Thorac
Cardiovasc Surg 1986;92:6-13. Cardiovasc Surg 1986;92:6-13.
Jobes DR, Nicolson SC, Steven JN, et al. Carbon dioxide jobes DR, Nicolson SC, Steven $J N$, et al. Carbon dioxide
prevents pulmonary overcirculation in hypoplastic left heart syndrome. Ann Thorac Surg 1992;54:150-1.

8 Helton JG, Aglira BA, Chin AJ, et al. Analysis of potential anatomic or physiologic determinants of outcome of palliative surgery for hypoplastic left heart syndrome. Circulation 1986;74:170-6.

9 Barber G, Helton JG, Aglira BA, et al. The significance of tricuspid regurgitation in hypoplastic left heart syndrome. Am Heart $f$ 1988;116:1563-7.

10 Barber G, Chin AJ, Murphy JD, Pigott JD, Norwood WI. Hypoplastic left heart syndrome: lack of correlation between pre-operative demographic and laboratory findings and survival following palliative surgery. Pediatr Cardiol 1989;10:129-34.

11 Rychik J, Murdison KA, Chin AJ, Norwood WI. Surgical management of severe outflow obstruction in lesions other than the hypoplastic left heart syndrome; use of a other than the hypoplastic left heart syndrome; use of a
pulmonary artery to aortic anastomosis. $\mathcal{F} \mathrm{Am}$ Coll pulmonary artery to aortic
Cardiol 1991;18:809-16.

12 Piggott JD, Murphy JD, Barber G, Norwood WI. Palliative reconstructive surgery for hypoplastic left heart syndrome. Ann Thorac Surg 1988;45:122-8.

13 Meliones JN, Snider AR, Bove EL, Rosenthal A, Rosen DA. Longitudinal results after first stage palliation for hypoplastic left heart syndrome. Circulation 1990;82 (suppl 5):IV 151-6.

14 Murdison KA, Baffa JM, Raffell PE, et al. Hypoplastic left heart syndrome. Outcome after initial reconstruction and before modified Fontan procedure. Circulation 1990;82 (suppl 5):IV 199-207.

15 Hebra A, Brown MF, Hirschl RB, et al. Mesenteric ischaemia in hypoplastic left heart syndrome. $\mathcal{F}$ Pediatr Surg 1993;28:606-11.

16 Norwood WI. Hypoplastic left heart syndrome. Ann Thorac Surg 1991;52:688-95.

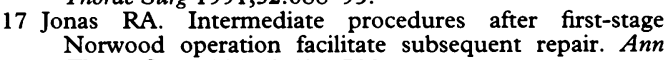
Thorac Surg 1991;52:696-700.

18 Farrell PE, Chang AC, Murdison KA, et al. Outcome and assessment after the modified Fontan procedure for 116-22. 